\title{
Automatic assignment of absolute configuration from 1D NMR data
}

\author{
Qing-You Zhang, Gonçalo Carrera, Mário J. S. Gomes, and João Aires-de-Sousa* \\ Departamento de Química, CQFB and REQUIMTE, Faculdade de Ciências e Tecnologia, \\ Universidade Nova de Lisboa, 2829-516 Monte de Caparica, Portugal \\ jas@fct.unl.pt
}

Received November 5, 2004

Opposite enantiomers exhibit different NMR properties in the presence of an external common chiral element, and a chiral molecule exhibits different NMR properties in the presence of external enantiomeric chiral elements. Automatic prediction of such differences, and comparison with experimental values, leads to the assignment of the absolute configuration. Here two cases are reported, one using a dataset of 80 chiral secondary alcohols esterified with (R)-MTPA and the corresponding $1 \mathrm{H}$ NMR chemical shifts and the other with 94 13C NMR chemical shifts of chiral secondary alcohols in two enantiomeric chiral solvents. For the first application, counterpropagation neural networks were trained to predict the sign of the difference between chemical shifts of opposite stereoisomers. The neural networks were trained to process the chirality code of the alcohol as the input, and to give the NMR property as the output. In the second application, similar neural networks were employed, but the property to predict was the difference of chemical shifts in the two enantiomeric solvents. For independent test sets of 20 objects, $100 \%$ correct predictions were obtained in both applications concerning the sign of the chemical shifts differences. Additionally, with the second dataset, the difference of chemical shifts in the two enantiomeric solvents was quantitatively predicted, yielding $r 20.936$ for the test set between the predicted and experimental values.

J. Org. Chem. 2005, 70, 2120-2130

10.1021/j0048029z CCC: $\$ 30.25 \odot 2005$ American Chemical Society

Published on Web 02/22/2005 